\title{
Fauna de flebotomíneos (Diptera: Psychodidae: Phlebotominae) em terra firme e planície fluvial na área de influência do gasoduto Coari-Manaus, Amazonas, Brasil
}

Luís Henrique Monteiro GOMES ${ }^{1}$, Liliane Coelho da Rocha NERY ${ }^{1}$, Francimeire Gomes PINHEIROํㅜ, Rui Alves FREITAS ${ }^{1}$, Antônia Maria Ramos FRANCO ${ }^{1}$

\section{RESUMO}

Este trabalho teve como objetivo realizar um levantamento preliminar da entomofauna de flebotomíneos nas áreas de influência do gasoduto Coari-Manaus (AM). As coletas foram realizadas no período de 4 a 8 de agosto (2003), em cinco dos sete municípios da área de influência do gasoduto, utilizando-se de armadilhas de luz do tipo CDC. Foram capturados 205 indivíduos, distribuídos em 25 espécies, pertencentes a oito subgêneros (151-73,7\%) e quatro grupos (54-26,3\%). A espécie Lutzomyia umbratilis foi a mais numerosa encontrando-se 21,0\% (43) do total coletado. Nestas coletas foi registrada pela primeira vez no Amazonas e segunda no Brasil a espécie Lutzomyia preclara, coletada nos municípios de Caapiranga e Manacapuru.

PALAVRAS-CHAVE: distribuição, Gasoduto, Leishmanioses, Lutzomyia umbratilis.

\section{Fauna of Sandflies (Diptera: Psychodidae: Phlebotominae) on terra firme and fluvial lowland in areas under the influence of the Coari-Manaus gas pipeline, Amazonas, Brazil}

\begin{abstract}
ABSTRAT
The object of this work was to make a preliminary survey of the entomofauna sandflies in the areas under the influence of the Coari-Manaus (AM) gas pipeline. The collections were made from 4 to 8 August 2003, in five of the seven municipal districts under the influence of the gas pipeline, using light traps of the type CDC. We captured 205 individuals, distributed in 25 species, belonging to eight subgenera $(151-73.7 \%)$ and four groups $(54-26.3 \%)$. The Lutzomyia umbratilis was the most numerous species found $(43-21.0 \%)$. The species Lutzomyia preclara, collected in the municipal districts of Caapiranga and Manacupuru, was registered for the first time in the state of Amazonas, and the second time in Brasil.
\end{abstract}

KEYWORDS: dstribution, Gas pipeline, Leishmaniasis, Lutzomyia umbratilis.

${ }^{1}$ Instituto Nacional de Pesquisas da Amazônia. E-mail: luishenriquemgomes@bol.com.br 
Os flebotomíneos (Diptera: Psychodidae: Phlebotominae) são insetos de importância médica por serem vetores de várias doenças, entre estas as leishmanioses. A diversidade das espécies na Amazônia resultou em uma considerável riqueza destes insetos, sendo quatro do subgênero Nyssomyia incriminadas na transmissão da doença: Lutzomyia (Nyssomyia) umbratilis Ward \& Fraiha, 1977 e L. (N.) anduzei Rozeboom, 1942, vetoras da Leishmania (Viannia) guyanensis Floch, 1954 (Lainson e Shaw, 1968; Arias e Freitas, 1978) e, $L$. (N.) flaviscutellata Mangabeira, 1942 e L. (N.) olmeca nociva Young \& Arias, 1982, vetoras da L. (Leishmania) amazonensis Lainson \& Shaw, 1972 (Lainson \& Shaw, 1968; Arias et al., 1987; RIMA, 2003).

No estado do Amazonas, a Petróleo Brasileiro S.A. (Petrobrás) explora jazidas de petróleo e gás natural na Bacia do Solimões, na província do rio Urucu, a $650 \mathrm{~km}$ de Manaus. Em 2003, esta empresa iniciou a construção de um gasoduto estimado em $397 \mathrm{~km}$ de extensão, com início em Coari finalizando em Manaus. No total, sete municípios serão direta ou indiretamente afetados. São eles: Coari, Codajás, Anori, Anamã, Caapiranga, Manacapuru e Iranduba. Sua área de influência direta abrange duas unidades geomorfológicas: terra firme e planície fluvial, compostas por vegetações ombrófilas de terras baixas - densas e abertas (RIMA, 2003).

$\mathrm{Na}$ implantação deste gasoduto, impactos em relação às leishmanioses poderão ocorrer quando do contato do homem com a floresta. Pois, segundo Araújo Filho (1981) e Talhari et al. (1988), no Brasil a história epidemiológica da Leishmaniose Tegumentar Americana (LTA) demonstra que os surtos epidêmicos da doença estão intimamente relacionados aos desmatamentos florestais, entre outros, acarretando a migração de mamíferos (reservatórios naturais de leishmânias) como, marsupiais (mucuras), edentados (tatus, preguiças e tamanduás) e roedores (ratos e camundongos) para outras áreas em busca de novos abrigos. Consequentemente, os flebotomíneos que antes realizavam seus repastos nesses animais, irão procurar suprir suas necessidades alimentares sugando o homem, que vai se fixar nestas áreas desmatadas, produzindo grandes epidemias da doença.

O objetivo deste trabalho foi realizar um levantamento preliminar da entomofauna de flebotomíneos nas áreas de influência da construção do gasoduto Coari-Manaus (AM).

Os flebotomíneos foram coletados no período de 4 a 8 de agosto de 2003, em cinco dos sete municípios envolvidos na rota de construção do gasoduto (Figura 1), com a utilização de quatro armadilhas luminosas do tipo CDC (CDC "miniature"- Hausherr Machine Works, New Jersey, EUA) em cada ponto de coleta. Os espécimes foram identificados segundo a chave taxonômica de Young e Duncan (1994).

Foram capturados 205 flebotomíneos, todos pertencentes ao gênero Lutzomyia França, 1924, distribuídos em 25 espécies,

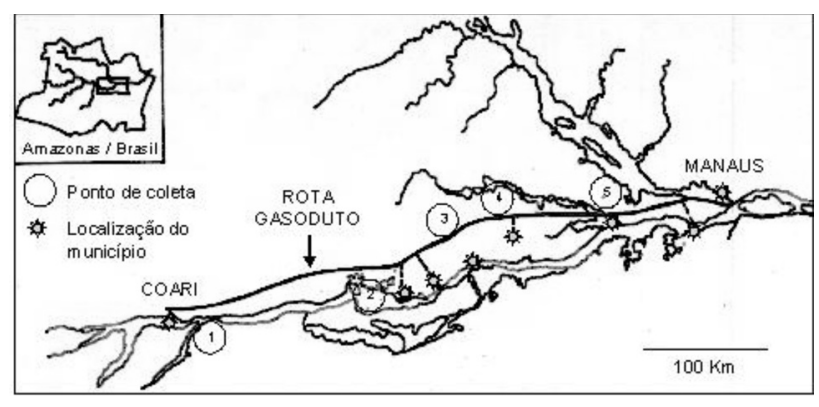

Figura 1 - Localização dos pontos de coletas de flebotomíneos na área de influência do gasoduto Coari-Manaus, Amazonas-Brasil (4 a 8 de agosto de 2003). (1) Coari (2) Codajás (3) Anamã (4) Caapiranga e (5) Manacapuru.

abrangendo oito subgêneros (151 - 73,7\%) e quatro grupos $(54-26,3 \%)$. Dentre estes, dois subgêneros apresentaram espécies vetoras de leishmaniose: (i) Nyssomyia - L. umbratrilis (43-21\%), L. flaviscutellata (12 - 5,9\%), L. anduzei $(10-$ $4,9 \%)$ e L. olmeca nociva $(2-1 \%)$ e (ii) Psychodopygus - $L$. davisi $(5-2,4 \%)$ e L. ayrozai $(1-0,5 \%)$ (Tabela 1$)$. Segundo, Grimaldi et al. (1991) e Gil et al. (2003), na Amazônia, as espécies destes subgêneros são comprovadamente importantes vetoras de leishmânias que causam leishmaniose cutânea, tanto no ciclo enzoótico, quanto no ciclo zoonótico.

Conforme a Tabela 1, o predomínio de L. umbratilis (43-21,7\%) chama atenção por se tratar de uma espécie considerada vetora potencial em florestas primárias de terra firme, sendo a principal transmissora da L. guyanensis (Arias e Freitas, 1977, 1978, 1982; Young e Duncan, 1994; e Cabanillas e Castellón, 1999). É bem provável que esta seja a espécie envolvida na maioria dos casos de leishmaniose oriundos das áreas de influência do Gasoduto como, Coari, Codajás, Manacapuru, Iranduba e Manaus (Rima, 2003).

Em áreas de planície fluvial deste empreendimento (Coari, Codajás e Caapiranga), os espécimes foram coletados em menor abundância ( $15-7,3 \%$ / Tabela 1$)$, devido à presença de muitos igarapés e a maioria por estar localizado em trechos de influência do gasoduto (Figura 1). Segundo o RIMA (2003), além dos igarapés é comum encontrar ao longo desta rota muitos lagos que são alinhados não somente pelas águas dos rios, mas também por igarapés de terra firme. Entretanto, nas capturas em ambiente de terra firme (Manacapuru e Anamã) os flebotomíneos foram mais numerosos (190 - 92,7\% / Tabela 1), certamente, pela maior disponibilidade de fonte alimentar (hospedeiros vertebrados), composta principalmente por roedores.

Neste estudo foi registrada pela primeira vez no Amazonas e a segunda no Brasil a ocorrência de Lu. (Sciopemyia) preclara Young e Arias, 1984, coletada nos municípios de Manacapuru e Caapiranga (Tabela 1). Esta espécie teve seu primeiro registro 
ACTA

AMAZONICA

Fauna de flebotomíneos (Diptera: Psychodidae: Phlebotominae) em terra firme e

planície fluvial na área de influência do gasoduto Coari-Manaus, Amazonas, Brasil

Tabela 1 - Número de flebotomíneos coletados na área de influência do gasoduto Coari-Manaus, Amazonas-Brasil (4 a 8 de agosto de 2003).

\begin{tabular}{|c|c|c|c|c|c|c|c|}
\hline \multirow{3}{*}{ Espécies } & \multicolumn{5}{|c|}{ Municípios } & \multirow{3}{*}{ Total } & \multirow{3}{*}{$\%$} \\
\hline & \multicolumn{3}{|c|}{ área de planície fluvial } & \multicolumn{2}{|c|}{ área de terra firme } & & \\
\hline & Coari & Codajás & Caapiranga & Anamã & Manacapuru & & \\
\hline \multicolumn{8}{|l|}{ Subgênero Nyssomyia } \\
\hline Lutzomyia umbratilis & 0 & 0 & 0 & 20 & 23 & 43 & 21,0 \\
\hline Lutzomyia reducta & 0 & 0 & 0 & 1 & 0 & 1 & 0,5 \\
\hline Lutzomyia olmeca nociva & 0 & 0 & 0 & 2 & 0 & 2 & 1,0 \\
\hline Lutzomyia flaviscutellata & 2 & 0 & 0 & 10 & 0 & 12 & 5,9 \\
\hline Lutzomyia antunesi & 1 & 0 & 0 & 0 & 37 & 38 & 18,5 \\
\hline Lutzomyia anduzei & 0 & 0 & 0 & 8 & 2 & 10 & 4,9 \\
\hline Subtotal & 3 & 0 & 0 & 41 & 62 & 106 & 51,7 \\
\hline \multicolumn{8}{|l|}{ Grupo Migonei } \\
\hline Lutzomyia walkeri & 0 & 0 & 1 & 0 & 38 & 39 & 19,0 \\
\hline Subtotal & 0 & 0 & 1 & 0 & 38 & 39 & 19,0 \\
\hline \multicolumn{8}{|l|}{ Subgênero Sciopemyia } \\
\hline Luzomyia preclara & 0 & 0 & 0 & 8 & 2 & 10 & 4,9 \\
\hline Lutzomyia sordellii & 0 & 0 & 0 & 2 & 1 & 3 & 1,5 \\
\hline Lutzomyia servulolimai & 0 & 0 & 0 & 1 & 0 & 1 & 0,5 \\
\hline Subtotal & 0 & 0 & 0 & 11 & 3 & 14 & 6,8 \\
\hline \multicolumn{8}{|l|}{ Subgênero Lutzomyia } \\
\hline Lutzomyia spatotrichia & 0 & 0 & 0 & 1 & 0 & 1 & 0,5 \\
\hline Lutzomyia gomezi & 0 & 0 & 0 & 0 & 9 & 9 & 4,4 \\
\hline Lutzomyia evangelistai & 0 & 0 & 0 & 0 & 2 & 2 & 1,0 \\
\hline Subtotal & 0 & 0 & 0 & 1 & 11 & 12 & 5,9 \\
\hline \multicolumn{8}{|l|}{ Grupo Oswaldoi } \\
\hline Lutzomyia rorotaensis & 0 & 0 & 1 & 0 & 6 & 7 & 3,4 \\
\hline Subtotal & 0 & 0 & 1 & 0 & 6 & 7 & 3,4 \\
\hline \multicolumn{8}{|l|}{ Grupo Saulensis } \\
\hline Lutzomyia saulensis & 0 & 2 & 3 & 0 & 2 & 7 & 3,4 \\
\hline Subtotal & 0 & 2 & 3 & 0 & 2 & 7 & 3,4 \\
\hline \multicolumn{8}{|l|}{ Subgênero Psychodopygus } \\
\hline Lutzomyia davisi & 0 & 0 & 1 & 4 & 0 & 5 & 2,4 \\
\hline Lutzomyia ayrozai & 0 & 0 & 0 & 1 & 0 & 1 & 0,5 \\
\hline Subtotal & 0 & 0 & 1 & 5 & 0 & 6 & 2,9 \\
\hline \multicolumn{8}{|l|}{ Subgênero Evandromyia } \\
\hline Lutzomyia monstruosa & 0 & 0 & 0 & 3 & 0 & 3 & 1,5 \\
\hline Lutzomyia georgii & 0 & 0 & 0 & 2 & 0 & 2 & 1,0 \\
\hline Subtotal & 0 & 0 & 0 & 5 & 0 & 5 & 2,4 \\
\hline \multicolumn{8}{|l|}{ Subgênero Trichophoromyia } \\
\hline Lutzomyia ubiquitalis & 0 & 1 & 0 & 0 & 0 & 1 & 0,5 \\
\hline Lutzomyia eurypyga & 1 & 0 & 0 & 0 & 0 & 1 & 0,5 \\
\hline Lutzomyia dunhami & 2 & 0 & 0 & 0 & 0 & 2 & 1,0 \\
\hline Subtotal & 3 & 1 & 0 & 0 & 0 & 4 & 2,0 \\
\hline \multicolumn{8}{|l|}{ Subgênero Psathyromyia } \\
\hline Lutzomyia cuzquena & 0 & 0 & 0 & 3 & 0 & 3 & 1,5 \\
\hline Subtotal & 0 & 0 & 0 & 3 & 0 & 3 & 1,5 \\
\hline \multicolumn{8}{|l|}{ Grupo Aragaoi } \\
\hline Lutzomyia aragaoi & 0 & 0 & 0 & 0 & 1 & 1 & 0,5 \\
\hline Subtotal & 0 & 0 & 0 & 0 & 1 & 1 & 0,5 \\
\hline
\end{tabular}


Tabela 1 - Continuação

\begin{tabular}{|c|c|c|c|c|c|c|c|}
\hline \multirow{3}{*}{ Espécies } & \multicolumn{5}{|c|}{ Municípios } & \multirow{3}{*}{ Total } & \multirow{3}{*}{$\%$} \\
\hline & \multicolumn{3}{|c|}{ área de planície fluvial } & \multicolumn{2}{|c|}{ área de terra firme } & & \\
\hline & Coari & Codajás & Caapiranga & Anamã & Manacapuru & & \\
\hline \multicolumn{8}{|l|}{ Subgênero Pintomyia } \\
\hline Lutzomyia damascenoi & 0 & 0 & 0 & 0 & 1 & 1 & 0,5 \\
\hline Subtotal & 0 & 0 & 0 & 0 & 1 & 1 & 0,5 \\
\hline Total & 6 & 3 & 6 & 66 & 124 & 205 & 100,0 \\
\hline$\%$ & 2,9 & 1,5 & 2,9 & 32,2 & 60,5 & 100,0 & \\
\hline
\end{tabular}

em 1987, na região da usina Hidrelétrica de Paredão/Roraima (comunicação pessoal, Freitas/2007, dados não publicados).

Segundo Arias \& Freitas (1978), o sistema fluvial do Rio Amazonas atua como barreira geográfica no ciclo vetor/ parasita/homem, possibilitando o encontro de outras espécies de flebotomíneos como prováveis vetores de leishmânias.

As informações acerca da distribuição de flebotomíneos na rota de influência do gasoduto, ainda são escassas. $\mathrm{O}$ levantamento de espécies de flebotomíneos em ambientes silvestres se faz necessário para indicar o predomínio de espécies vetores de leishmanioses, em áreas onde o homem esteja exercendo alguma atividade, principalmente nas áreas de influência da construção do gasoduto, o que consequentemente levará a um aumento do fluxo migratório de população susceptível atraídas pela construção do empreendimento, a ocorrência de desmatamento e outras alteraçôes ambientais que deverão contribuir para que haja uma maior exposição aos vetores da doença, levando a um incremento na freqüência de LTA na região, especialmente na população de trabalhadores locais.

\section{BIBLIOGRAFIA CITADA}

Araújo Filho, N.A 1981. Leishmaniose Tegumentar Americana e o desmatamento da Amazônia. Acta Amazônica,11(1): 87-189.

Arias, J.R.; Freitas, R.A. 1977. On the vector of cutaneous leishmaniasis in the Central Amazon of Brazil. Acta Amazônica, 7: 293.

Arias, J.R.; Freitas, R.A. 1978. Sobre os vetores de leishmaniose cutânea na Amazônia Central do Brasil. 2: Incidência de flagelados em flebótomos selváticos. Acta Amazônica, 8: 387 396.

Arias, J.R.; Freitas, R.A. 1982. On the vectors of cutaneous leismaniases in Central Amazon of Brazil. 3. Phlebotomine sandfly stratificacion in a terra firme Forest. Acta Amazônica. 12: 599-603.
Arias, J.R.; Freitas, R.A.; Naiff, R.D.; Barrett, T.V. 1987. Observation on the parasite Leishmania mexicana amazonensis and its natural infection of the sandfly Lutzomyia olmeca nociva. PAHO Bulletin 21(1): 48-54.

Cabanillas, M.R.S.; Castellón, E.G., 1999. Distribuition of sandflies (Diptera: Psychodidae) on tree-trunks in a non-flooded area of Ducke Forest Reserve, Manaus, Am, Brazil. Mem. Inst. Oswaldo Cruz, 94: 289-296.

Gil, L.H.S.; Basano, S.A.; Souza, A.A.; Silva, M.G.S.; Barata, I.; Ishikawa, E. A.; Camargo, L.M.A.; Shavv, J.J. 2003. Recent observations on the sand fly (Diptera: Psychodidae) fauna of the State of Rondónia, Western Amazónia, Brazil: The importance of Psychodopyigus davisi as a vector of zoonotic cutaneous leishmaniasis. Mem. Inst. Oswaldo Cruz, 98: 751-755.

Girimaldi Jr, G.; Momen, H,; Naiff, R.D.; Mcmhon-Pratt, D.; Barrett, T.V. 1991. Caracterization and classification of leishmanial parasites from humans, wild mammals., and sand flies in the Amazon Region of Brazil. Am. J. Trop. Med. Hyg., 44: 645-661.

Lainson, R.; Shaw, J.J. 1968. Leishmaniasis in Brazil: 1. Observation on enzootic rodent leishmaniasis - incrimination of Lutzomyia flaviscutellata (Mangabeira) as the vector in the lower Amazonian basin. Trans. Roy. Soc. Trop. Med. Hyg., 62(3): 396-405.

RIMA, Relatório de Impactos Ambientais 2003. Gasoduto CoariManaus, Amazonas. 249pp.

Talhari, S.; Arias, J.A.; Cunha, M.G.S.; Naiff, R.D.; Naiff, M.F.; Freitas, R.A.; Barrett, T., 1988. Leishmaniose no Estado do Amazonas-Aspectos epidemiológicos, clínicos e terapêuticos. An. Bras. Dermatol., 63(6): 433-438.

Young, D.G.; Duncan, M.A. 1994. Guide to the identification and geographic distribution of Lutzomyia sand flies in Mexico, the West Indies, Central and South America (Diptera: Psychodidae). Memoirs of the American Entomological Institute, no 54, Associated Publishers, Gainesville, 881pp.

Recebido em 28/01/2008

Aceito em 28/04/2008 